\title{
ULTRA-SENSITIVE OPTO-PIEZORESISTIVE SENSORS UTILISING 3C-SIC/SI HETEROSTRUCTURES
}

\author{
Thanh Nguyen ${ }^{1 *}$, Toan Dinh ${ }^{1}$, Abu Riduan Md Foisal ${ }^{1}$, Hoang-Phuong Phan ${ }^{1}$, Tuan-Khoa Nguyen ${ }^{1}$, \\ Nam-Trung Nguyen ${ }^{1}$ and Dzung Viet Dao ${ }^{1,2}$ \\ ${ }^{1}$ Queensland Micro and Nanotechnology Centre, Griffith University, Queensland, AUSTRALIA and \\ ${ }^{2}$ School of Engineering and Built Environment, Griffith University, Queensland, AUSTRALIA
}

\begin{abstract}
Here we report for the first time an ultra-sensitive opto-piezoresistive effect in cubic silicon carbide (3C$\mathrm{SiC})$ nanofilms grown on silicon $(\mathrm{Si})$. The sensitivity of the sensor was significantly enhanced by coupling the photovoltaic effect and controlling distribution of hole/electron in semiconductors. By applying this method, the gauge factor (GF) of strain sensors can be improved at least three orders of magnitude compared to conventional MEMS sensor. A GF of approximately 58,000 was observed, which is the highest GF reported for semiconductor piezoresistive sensors to date. Consequently, our findings can be deployed to develop ultra-sensitive mechanical sensors and MEMS/NEMS sensing applications.
\end{abstract}

\section{KEYWORDS}

Piezoresistance, piezoresistive effect, optopiezoresistive, heterojunctions, ultra-sensitive strain sensor, photovoltaic effect.

\section{INTRODUCTION}

Several physical sensing concepts have been employed to develop microsensors and flexible electronic devices, including piezoresistance and thermoresistance [1-3]. Among them, the piezoresistive effect in semiconductors have been widely utilised in sensor applications, such as pressure, force, tactile and strain sensors, accelerometers, and microphones owing to its simplicity in design and fabrication [4-8]. However, the sensitivity of piezoresistive-based sensor is limited by the nature of the sensor materials.

It is of high interest to discover a novel sensing approach to boost the sensitivity of piezoresistance sensors. Conventional methods have been investigated, including arrangement of piezoresistors in optimal crystal orientations [9], optimization of carrier concentrations, or selection of materials with high piezoresistive coefficients. While $\mathrm{Si}$ is considered as the most suitable material for mechanical sensor applications with high piezoresistive coefficients and the development of fabrication technology based on $\mathrm{Si}, \mathrm{SiC}$ is emerging as one of the best materials for harsh environment utilisations [10]. However, the gauge factor is typically smaller than 200 [11].

Additionally, miniaturing piezoresitors to nanoscale also has been conducted owing to the advance of nanofabrication techniques [12-14] . A gauge factor of up to 1547 was achieved for the silicon nanowires [12]. However, this approach is still controversial [10, 15-17].
Modulation of charge carriers to achieve a high GF has emerged as a promising approach for significant improvement of sensitivity of mechanical sensors. For instance, by using an external bias voltage to modulate an electric generated field, Neuzil et al. [18] have successfully demonstrated a giant piezoresistive effect in Si nanowire with a GF of up to 5,000.

Herein we propose an ultra-sensitive optopiezoresistive sensors with $3 \mathrm{C}-\mathrm{SiC} / \mathrm{Si}$ heterojunction structure. The sensor is a $3 \mathrm{C}-\mathrm{SiC} / \mathrm{Si}$ heterojunction cantilever in which the sensing element (piezoresistor) is formed by patterning aluminum on the top of $3 \mathrm{C}$-SiC thin film. While the piezoresistive effect is used to detect change of resistance corresponding with the change of load, the coupling of photovoltaic effect and controlling external electric field is utilized to tune the performance of the piezoresistive effect, resulting in modulating the sensitivity of the sensor. The sensing element is illuminated by a non-uniform visible light and the tuning current optimally is controlled by a source meter. By using this method, the GF of the strain sensor can be achieved as high as 58,000 which is the highest GF reported to date.

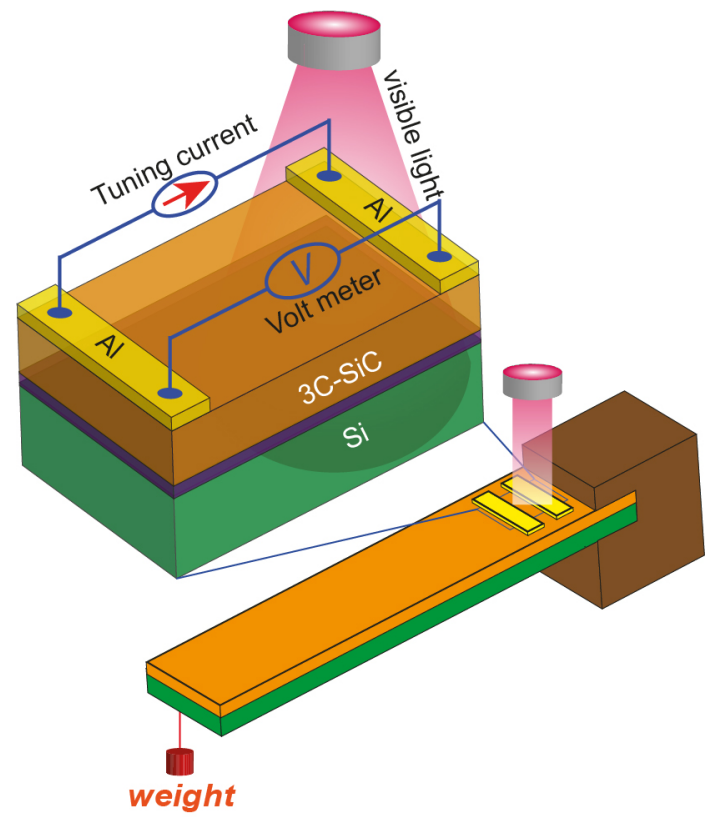

Figure 1: Operating principle of the opto-piezoresistive strain sensor. The sensor is a 3C-SiC/Si heterojunction cantilever in which two aluminum electrodes are formed on the top of $3 \mathrm{C}$-SiC thin film through a patterning process. While the piezoresistive effect is used to detect change of resistance versus change of load, illuminating non-uniform visible light on the sensing element coupled 
with controlling accurately supplied current are utilised to modulate the sensitivity of the sensor.<smiles>[GeH3]</smiles>

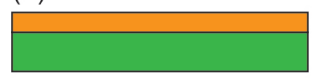

(2)

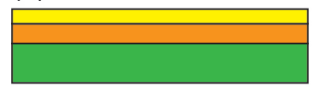

(3)

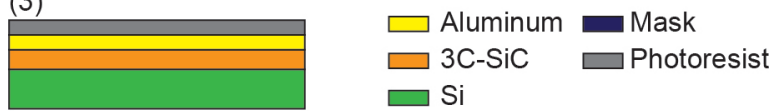

Figure 2: Device fabrication process. 3C-SiC thin film was grown on (100) p-type single crystalline Si Substrate. The 3C-SiC/Si wafer, then, was diced to create a cantilever structure. Finally, two aluminum electrodes were patterned on the top of the $3 \mathrm{C}$-SiC thin film.

\section{METHODS}

\section{Principle of operation}

As depicted in Figure 1, the strain sensor is a cantilever structured by two material layers to create a $3 \mathrm{C}-\mathrm{SiC} / \mathrm{Si}$ heterojunction. One end of the cantilever is firmly clamped, while the other free end receives external force resulting in strain in the cantilever. In this prototype, the sensing element near the clamped end of cantilever is illuminated by a non-uniform visible light. A source meter is used to supply the optimal current and simultaneously collect voltage data.

\section{Table 1: Sample parameters}

\begin{tabular}{|l|c|c|}
\hline Parameters & Top layer & Substrate \\
\hline Materials & $\mathrm{p}^{+}-3 \mathrm{C}-\mathrm{SiC}$ & $\mathrm{p}-\mathrm{Si}$ \\
\hline Thickness & $380 \mathrm{~nm}$ & $0.63 \mathrm{~mm}$ \\
\hline Doped concentrations & $5 \cdot 10^{18} \mathrm{~cm}^{-3}$ & $5 \cdot 10^{14} \mathrm{~cm}^{-3}$ \\
\hline
\end{tabular}

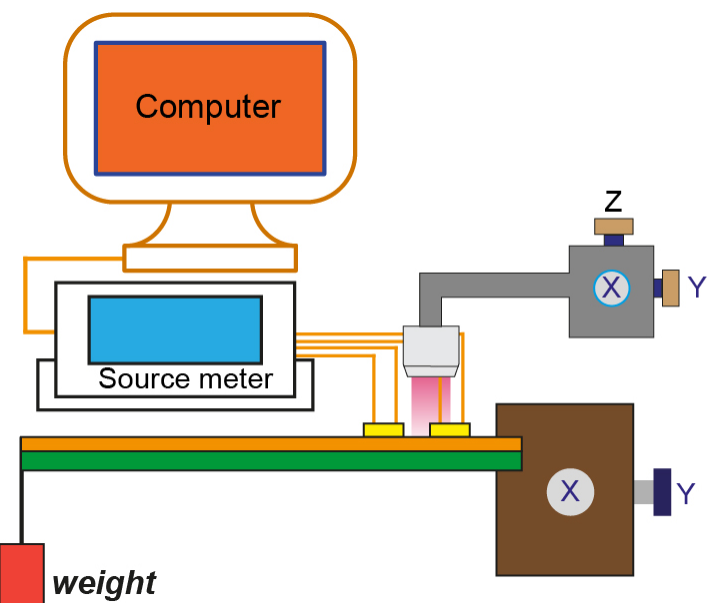

Figure 3: Experimental setup. One end of the cantilever was firmly clamped, and different loads were exerted on the free end to induce strain in the cantilever. The light beam position was precisely adjusted in three directions. A KEITHLEY 2450 Source Meter was used to control the supply current and measure voltage at the same time.

\section{Device fabrication}

Single crystalline heavily doped $3 \mathrm{C}-\mathrm{SiC}$ with a thickness of $380 \mathrm{~nm}$ was grown on a p-type Si (100) substrate by a low pressure chemical deposition (LPCVD) method at a temperature of $1,000^{\circ} \mathrm{C}$ (Figure 2). Then, aluminum was deposited on the surface of the $3 \mathrm{C}-\mathrm{SiC}$ thin film. Finally, the two aluminum electrodes were patterned through a lithography process, followed by an annealing process at $800^{\circ} \mathrm{C}$ to create Ohmic contact between aluminum and $3 \mathrm{C}-\mathrm{SiC}$ thin film.

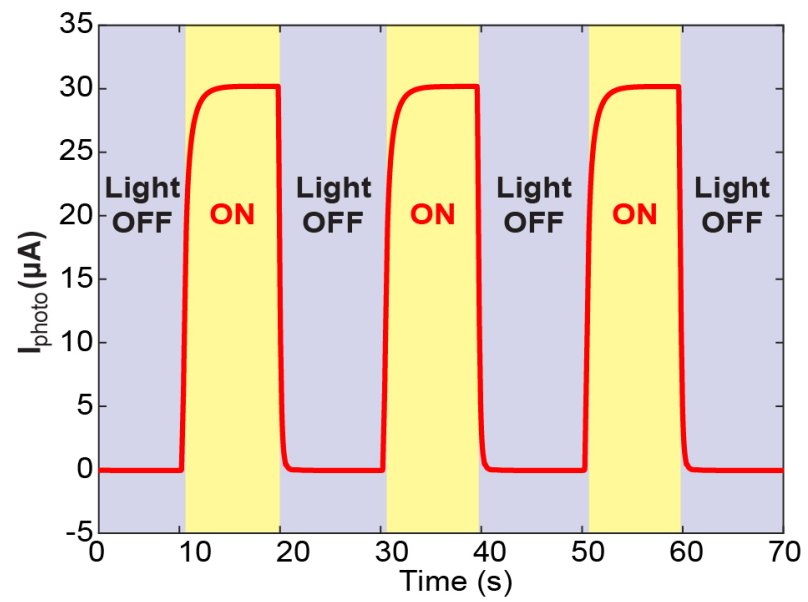

Figure 4: The repeatability of the photo current. The light intensity and the light position are adjusted so that the generated voltage and photo current were approximately $9 \mathrm{mV}$ and $29.75 \mu \mathrm{A}$, respectively.

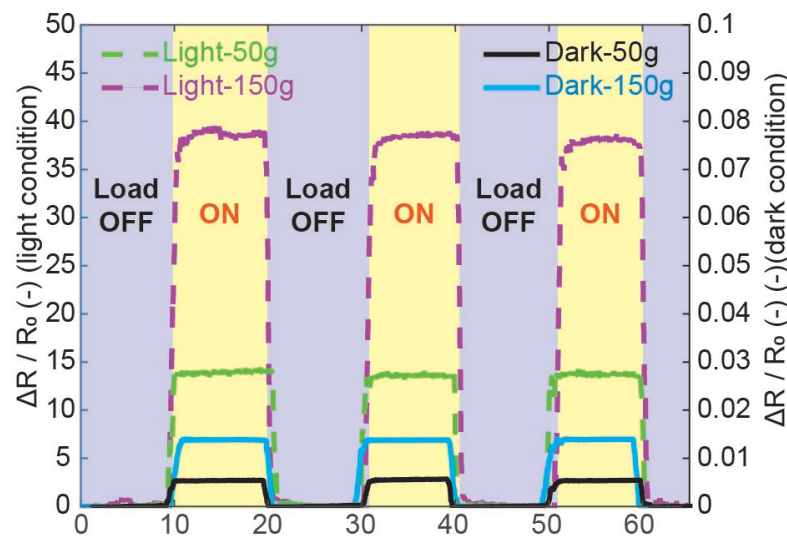

Figure 5: Repeatability of relative change of output voltage at different loads. Two loads of $50 \mathrm{~g}$ and $150 \mathrm{~g}$ were applied and released periodically. The supply current was kept at optimal value of $29.75 \mu \mathrm{A}$, and the voltage between two electrodes was measured under light and dark conditions. Fractional change of resistance under the light condition is much higher than that under the dark condition.

\section{Device parameters and Experiment}

As depicted in Table 1, the carrier concentrations in $3 \mathrm{C}-\mathrm{SiC}$ thin film and $\mathrm{Si}$ substrate are respectively $5 \times 10^{18}$ $\mathrm{cm}^{-3}$ and $5 \times 10^{14} \mathrm{~cm}^{-3}$ which were measured by the hot probe and Hall effect techniques. As shown in Figure 3, to investigate the performance of the strain sensor, one end of the cantilever was firmly clamped, and the other end was the position where the load was applied. The sensing element was illuminated by a non-uniform visible light, and the tuning current was precisely controlled using a source meter. The light beam was accurately positioned in 
three dimensions so that the generated voltage and the photo current were approximately $9 \mathrm{mV}$ and $29.8 \mu \mathrm{A}$. Figure 4 shows the repeatability of the photocurrent after adjusting the light position and the light intensity when the light was periodically turned ON and OFF. While the photocurrent was about $29.75 \mu \mathrm{A}$ under light condition, the figure was about $0 \mu \mathrm{A}$ under dark condition. The optimal current used to tune the sensitivity of the sensor was around the magnitude of the photocurrent $(I=29.75$ $\mu \mathrm{A})$. Two different loads of $50 \mathrm{~g}$ and $150 \mathrm{~g}$ were used to induce strain in the sensor.

\section{RESULTS AND DISCUSSION}

Figure 5 shows the repeatability of relative change of resistance when two different loads of $50 \mathrm{~g}$ and $150 \mathrm{~g}$ were applied and released periodically under light and dark conditions. It is seen that under the light condition the relative change of resistance is about 14 and 38 corresponding with loads of $50 \mathrm{~g}$ and $100 \mathrm{~g}$, while these values are approximately 0.005 and 0.0138 , respectively, under the dark condition. By simultaneously controlling light illumination and electric field in the 3C-SiC layer, the relative change of the resistance under light illumination is at least three orders of magnitude higher than that in dark condition.

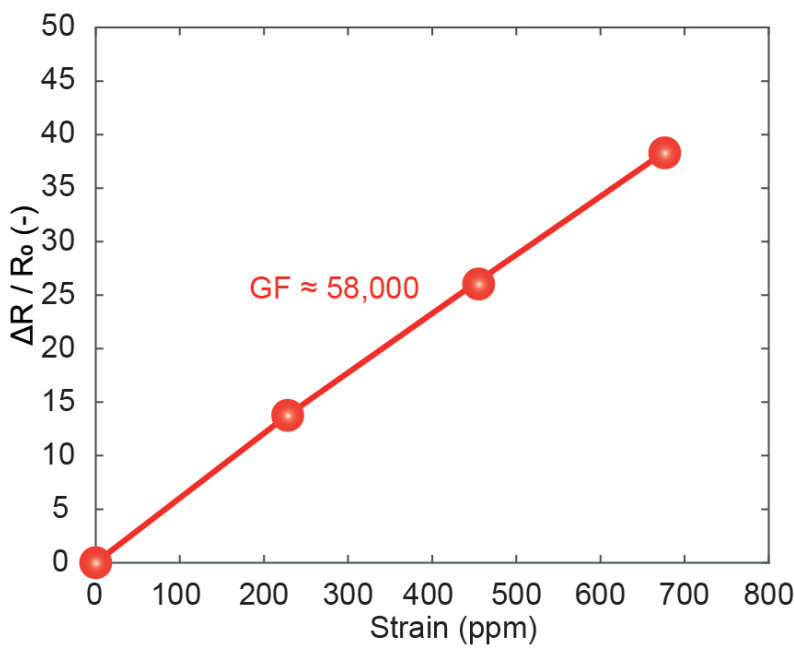

Figure 6. The sensitivity of the strain sensor. By coupling photovoltaic effect and tuning current, the GF of the optopiezoresistive strain sensor was modulated to as high as 58,000 which is the highest GF reported to date.

Moreover, the linearity of the sensor is excellent.

The GF, a parameter used to evaluate the sensitivity of a strain sensor, is defined as relative change of the resistance by the applied strain $\mathrm{GF}=(\Delta \mathrm{R} / \mathrm{R}) \cdot(1 / \varepsilon)$. By coupling the photovoltaic effect with controlling the supply current, the GF of the opto-piezoresistive sensor can achieve as high as approximately 58,000 (Figure 6), which is the highest GF ever reported to date. This value is much higher than the GF of currently commercialized strain sensors. It also is seen in Figure 6 that the resistance changed linearly with increasing strain which is desirable for strain sensing applications.

Figure 7 (a) shows the band diagram of the $3 \mathrm{C}-\mathrm{SiC} / \mathrm{Si}$ heterojunction, evidencing the generation of electron-hole pairs and charge transportation in $3 \mathrm{C}-\mathrm{SiC} / \mathrm{Si}$ under light illumination. Photons with different wavelengths injected to the sensing element areas are absorbed primarily in the depletion region of heterojunction and in Si substrate, resulting in the generation of electron hole pairs (EHPs) in these regions. Generated holes are then injected into the $3 \mathrm{C}-\mathrm{SiC}$ thin film thanks to the internal field in depletion region and tunneling mechanism while generated electrons move in the reverse direction. Consequently, the holes and electrons are redistributed in $3 \mathrm{C}-\mathrm{SiC}$ and $\mathrm{Si}$ layers. Under non-uniform light intensity, an electric field is formed between the two electrodes which is measured as photovoltage. By supplying an optimally controlled electric current, the voltage between the two electrodes can approach zero under a free-strain condition.

Figure 7 (b) depicts the shift of valance bands of heavy holes $(\mathrm{HH})$ and light holes $(\mathrm{LH})$ under tensile stress to explain the repopulation and mobility change (and therefore, resistivity change) of the charge carriers in C3$\mathrm{SiC}$ and $\mathrm{Si}$ [19]. When load is applied to the sensor, energy bands of $\mathrm{HH}$ and $\mathrm{LH}$ in $3 \mathrm{C}-\mathrm{SiC}$ are shifted. Consequently, concentrations of $\mathrm{HH}$ and $\mathrm{LH}$ change, resulting in the change of hole mobility which increases resistance. So, the significant enhancement of the strain sensing performance is attributed to the photoexcitation of charge carriers, as well as the strain-modulated mobility change of charge carriers in $\mathrm{SiC}$.
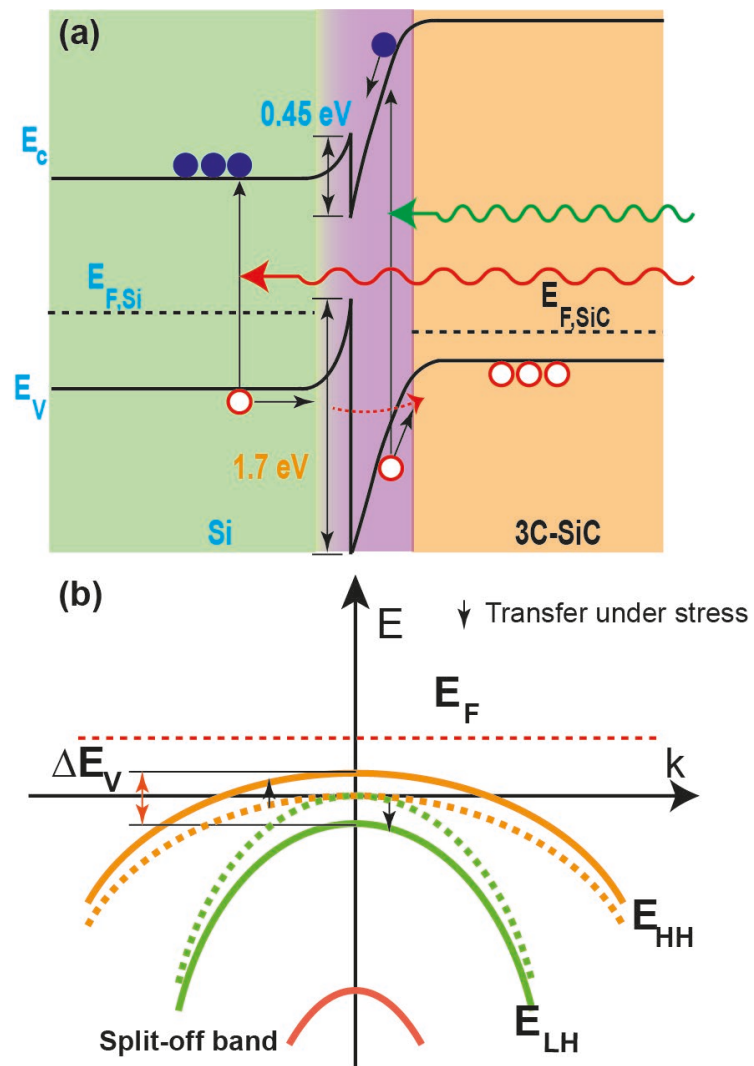

Figure 7. (a) Band energy and (b) E-k characteristic in $3 C$-Si thin film.

\section{CONCLUSIONS}

We successfully demonstrated an ultra-sensitive optopiezoresistive strain sensor with a giant GF of 58,000. This is the highest GF reported to date and much higher than that of commercial strain sensors. The sensitivity of 
the sensor was enhanced by combining the effect of nonuniform light illumination and the effect of externally controlled electric field. The EHPs are generated and redistributed under light illumination resulting in a lateral photovoltage. An electric field created by tuning current then compensates the photovoltage under free-strain condition. The strain results in the shift of the energy band of HHs and LHs, hence increases the resistance.

\section{ACKNOWLEDGEMENTS}

The 3C-SiC material was developed and supplied by Leonie Hold and Alan Iacopi of the Queensland Microtechnology Facility, part of the Queensland node Griffith - of the Australian National Fabrication Facility. A company established under the National Collaborative Research Infrastructure Strategy to provide nano and microfabrication facilities for Australia's researchers. The epitaxial SiC deposition was developed as part of Griffith Universities Joint Development Agreement with SPT Microtechnology, the manufacturer of the Epiflx production reactor. This work has been partially supported by Australian Research Council grants LP150100153 and LP160101553. T. Dinh is grateful for the support from Griffith University/Simon Fraser University Collaborative Travel Grant2017 and Griffith University New Research Grant 2019.

\section{REFERENCES}

[1] H.-P. Phan et al., "Nano strain-amplifier: Making ultra-sensitive piezoresistance in nanowires possible without the need of quantum and surface charge effects," Applied Physics Letters, vol. 109, no. 12, p. 123502, 2016.

[2] T. Dinh, H.-P. Phan, A. Qamar, N.-T. Nguyen, and D. V. Dao, "Flexible and multifunctional electronics fabricated by a solvent-free and userfriendly method," RSC Advances, vol. 6, no. 81, pp. 77267-77274, 2016.

[3] T. Dinh et al., "Solvent-free fabrication of biodegradable hot-film flow sensor for noninvasive respiratory monitoring," Journal of Physics D: Applied Physics, vol. 50, no. 21, p. 215401, 2017.

[4] H.-P. Phan et al., "Piezoresistive effect in p-type $3 \mathrm{C}-\mathrm{SiC}$ at high temperatures characterized using Joule heating," Scientific reports, vol. 6, p. 28499 , 2016.

[5] M. A. S. M. Haniff et al., "Piezoresistive effects in controllable defective HFTCVD graphene-based flexible pressure sensor," Scientific reports, vol. 5, p. $14751,2015$.

[6] T.-K. Nguyen et al., "Highly sensitive 4H-SiC pressure sensor at cryogenic and elevated temperatures," Materials \& Design, vol. 156, pp. 441-445, 2018.

[7] H.-P. Phan et al., "Highly sensitive pressure sensors employing 3C-SiC nanowires fabricated on a free standing structure," Materials \& Design, vol. 156, pp. 16-21, 2018.

[8] T. Yamada et al., "A stretchable carbon nanotube strain sensor for human-motion detection," Nature nanotechnology, vol. 6, no. 5, p. 296, 2011.
[9] Y. Kanda, "A graphical representation of the piezoresistance coefficients in silicon," IEEE Transactions on electron devices, vol. 29, no. 1, pp. 64-70, 1982.

[10] A. R. Md Foisal et al., "Pushing the Limits of Piezoresistive Effect by Optomechanical Coupling in $3 \mathrm{C}-\mathrm{SiC} / \mathrm{Si}$ Heterostructure," ACS applied materials \& interfaces, vol. 9, no. 46, pp. 3992139925, 2017.

[11] A. A. Barlian, W.-T. Park, J. R. Mallon, A. J. Rastegar, and B. L. Pruitt, "Semiconductor piezoresistance for microsystems," Proceedings of the IEEE, vol. 97, no. 3, pp. 513-552, 2009.

[12] K. Reck, J. Richter, O. Hansen, and E. V. Thomsen, "Piezoresistive effect in top-down fabricated silicon nanowires," in Proc. IEEE MEMS, 2008, pp. 717-720.

[13] A. Koumela et al., "Piezoresistance of top-down suspended Si nanowires," Nanotechnology, vol. 22, no. 39, p. 395701, 2011.

[14] R. He and P. Yang, "Giant piezoresistance effect in silicon nanowires," Nature nanotechnology, vol. 1, no. 1, p. 42, 2006.

[15] J. Milne, A. Rowe, S. Arscott, and C. Renner, "Giant piezoresistance effects in silicon nanowires and microwires," Physical review letters, vol. 105, no. 22, p. 226802, 2010.

[16] A. Rowe, "Piezoresistance in silicon and its nanostructures," Journal of Materials Research, vol. 29, no. 6, pp. 731-744, 2014.

[17] H.-P. Phan et al., "The piezoresistive effect in topdown fabricated p-type 3C-SiC nanowires," IEEE Electron Device Letters, vol. 37, no. 8, pp. 10291032, 2016.

[18] P. Neuzil, C. C. Wong, and J. Reboud, "Electrically controlled giant piezoresistance in silicon nanowires," Nano letters, vol. 10, no. 4, pp. 1248-1252, 2010.

[19] A. Qamar, P. Tanner, D. V. Dao, H.-P. Phan, and T. Dinh, "Electrical properties of p-type 3C-SiC/Si heterojunction diode under mechanical stress," IEEE Electron Device Letters, vol. 35, no. 12, pp. 1293-1295, 2014.

\section{CONTACT}

*Thanh Nguyen, tel: +61479148686; thanh.nguyen11@griffithuni.edu.au 